\section{Cahiers de Narratologie}

Analyse et théorie narratives

10.2 | 2001

La voix narrative

\title{
Voix de l'ironie et voix du fantastique dans la nouvelle du XIXe siècle
}

Peggy Karpouzou

\section{(2) OpenEdition}

1 Journals

Édition électronique

URL : http://journals.openedition.org/narratologie/10186

DOI : 10.4000/narratologie.10186

ISSN : 1765-307X

Éditeur

LIRCES

Édition imprimée

Date de publication : 1 janvier 2001

Pagination : 91-103

ISBN : 2914561032

ISSN : $0993-8516$

Référence électronique

Peggy Karpouzou, «Voix de l'ironie et voix du fantastique dans la nouvelle du XIXe siècle », Cahiers de Narratologie [En ligne], 10.2 | 2001, mis en ligne le 01 janvier 2001, consulté le 11 juin 2020. URL

http://journals.openedition.org/narratologie/10186; DOI : https://doi.org/10.4000/narratologie.10186 


\title{
VOIX DE L'IRONIE ET VOIX DU FANTASTIQUE DANS LA NOUVELLE DU XIX ${ }^{\mathrm{e}}$ SIÈCLE
}

\author{
Peggy KARPOUZOU \\ Université de la Sorbonne Nouvelle (PARIS III)
}

L'homme ne cède aux anges et ne se rend entièrement à la mort que par l'infirmité de sa pauvre volonté1. Cette affirmation grandiloquente de l'épigraphe de «Ligeia» de Poe sollicitant l'adhésion du lecteur pourrait introduire à un récit fantastique; ou avec un clin d'œil moqueur, à un récit ironique - comique. Est-ce qu'elle pourrait avoir un double statut, à la fois fantastique et ironique ? Nous allons aborder le rapport entre ironie et fantastique à partir de deux nouvelles classiques du XIX' siècle, « Véra » de Villiers de l'Isle-Adam et «Ligeia » de E. A. Poe, traitant toutes deux un thème typique du fantastique, celui de "la morte-vivante». Plus particulièrement, en exploitant l'hypothèse de $\mathrm{Ph}$. Hamon sur la connivence du fantastique et de l'ironique ${ }^{2}$, on étudiera les affinités structurelles et idéologiques entre fantastique et ironique et leur coexistence au sein du même texte.

"Véra " nous fournit un exemple intéressant. Même après la mort et l'enterrement de sa femme Véra, le comte d'Athol continue à vivre comme si elle était encore vivante ; cette mise en scène hallucinatoire aboutit à l'ironie du fait de la mort de Véra : Le jour de sa fête, il plaça, par plaisanterie, une immortelle dans le bouquet qu'il jeta sur l'oreiller de Véra. - «Puisqu'elle se croit morte », dit il ${ }^{3}$.

${ }^{1}$ Edgar Allan Poe : Contes-Essais-Poèmes, C. Richard (ed), Paris, Robert Laffont, coll. «Bouquins », 19994, p. 362.

${ }^{2} \mathrm{Ph}$. HAMON, L'ironie littéraire : Essai sur les formes de l'écriture oblique, Paris, Hachette, 1996, pp. 57-9.

${ }^{3}$ Villiers de l'Isle-Adam, Euvres complètes, t. I, A. Raitt - P. G. Castex (ed), Paris, Gallimard, Bibliothèque de la Pléiade, 1986, p. 558. 
Le comte d'Athol, tenant à la conviction que "Véra n'est pas morte », ironise sur la prétendue pensée de sa femme, qui serait « je suis morte "; évidemment cet énoncé présupposé pris littéralement est impossible, tout comme son énonciation ; ils sont paradoxaux, menant à une "affirmationnégation », « je suis morte et pas morte », l'affirmation « je suis morte » constituant en même temps sa négation ${ }^{4}$. Cette même phrase paradoxale se trouve au cœur et à l'origine du surnaturel, celui qui provoque l'apparition de Véra et fait d'elle une morte-vivante ; elle condense ainsi la jonction entre fantastique et ironique. D'ailleurs la fin du surnaturel de l'apparition se réalise par l'affirmation opposée et nullement ironique du comte : - Ah! maintenant, je me rappelle!...dit il. Qu'ai-je donc? - Mais tu es morte! (ibid., p. 560), qui cause l'évanescence de Véra. Entre ces deux phrases, l'ironique "Puisqu'elle se croit morte » et la non-ironique «Mais tu es morte!" se situe l'engendrement et la destruction du phénomène surnaturel. Ainsi le fantastique de la «morte-vivante » apparaît comme un fait de langage, et même compatible avec le discours ironique. Pourrait-on parler alors d'une affinité entre discours fantastique et discours ironique?

\section{Affinités structurelles et idéologiques : approche tensionnelle}

\section{Une structure double et antithétique}

Nous considérons que la notion de la tension est la plus appropriée pour une définition commune des deux discours et nous introduirons ici une analyse tensionnelle. L'ironie " comme trope », selon l'approche rhétorique traditionnelle, ou « comme mention", selon l'approche de Sperber - Wilson qui a été le fondement des approches polyphoniques

${ }^{4}$ Cf. l'analyse du paradoxe linguistique de la phrase : "Il disait maintenant : - Oui, - non, - j'ai dormi, - et maintenant, je suis mort » dans le conte "The Facts in the Case of M. Valdemar " de Poe, où il ne s'agit point d'un énoncé incroyable, mais plus radicalement d'une énonciation impossible selon R. Barthes, "Analyse textuelle d'un conte d'Edgar Poe " in L'aventure sémiologique, Paris, Seuil, 1985, pp. 351-4. 
récentes, est toujours un discours à deux niveaux et paradoxal ${ }^{5}$; cette structure résulte d'une tension entre des éléments co-présents et incongrus dans un contexte donné, comme dans notre exemple l'affirmation paradoxale "Puisqu'elle se croit morte» précédée par la phrase "il plaça par plaisanterie, une immortelle dans le bouquet".

Plus en détail, l'ironie du point de vue sémantique est définie par sa structure double et antithétique, c'est-à-dire à la fois citationnelle du premier niveau [thèse] et polémique de celui-ci [anti-thèse] ; du point de vue pragmatique, elle est définie par la fonction critique fondamentalement négative de cette tension, ce qui lie l'ironie aux systèmes de normes et de valeurs ${ }^{6}$. Ainsi au premier niveau l'énonciateur prétend adhérer au discours cité/présupposé " je suis morte », mais en même temps, dans un second niveau co-présent dans l'énonciation, le discours engendré par la connotation pragmatique de négativité, inhérente dans l'ironie, disqualifie le discours du premier.

L'activation de cette tension passe à travers un système signalétique de marques et la construction d' "une scène énonciative » à quatre actants : l' "ironiste ", créateur du discours ironique, (ici le comte d'Athol), l'interprète de l'ironie (le lecteur, qui peut être un «co-ironiste» ou un " non - ironiste ») et l' « ironisé », la cible du discours ironique (Véra dans notre exemple); dans la forme la plus abstraite du terme, on propose la conception de l'ironisé en tant que norme jugée comme "norme d'impertinence » (en l'occur-

${ }^{5}$ Cf. les articles classiques de C. Kerbrat - Orecchioni, «L'ironie comme trope", Poétique 41,1980, pp. 108-27 et D. Sperber D. Wilson, "Les ironies comme mentions", Poétique 36, 1978, pp. 399-412. Pour un compte rendu des approches rhétoriques linguistiques de l'ironie, cf. L. Perrin, L'Ironie mise en trope, Paris, Kimé, 1996.

${ }^{6} \mathrm{Ce}$ rattachement de l'ironie aux normes et aux valeurs la ramène à l'ironie socratique, mode de conversation particulier de Socrate où il s'agit de faire sortir des opinions préconçues, le général, le concept, l'objectif, cf. E. Behler, Ironie et Modernité, Paris, PUF, 1996, p. 179. Pour $\mathrm{Ph}$. Hamon, le dédoublement axiologique prime sur le dédoublement sémantique, op. cit., 1996, pp. 30 sqq., position qu'on adopte aussi en considérant la fonction axiologique comme trait distinctif de l'ironie par rapport autres phénomènes d'ambiguïté. 
rence, la conviction « je suis morte ») par rapport au quatrième actant de la communication ironique, que nous nommons la 《 norme de pertinence ${ }^{7}$, impliquée par le discours ironique (ici c'est la conviction que «Véra n'est pas morte »).

Nous faisons une distinction opérationnelle entre deux formes d'ironie, selon la résolution ou non de la tension: l'ironie qui perpétue la tension de façon indécidable est une ironie tensionnelle, équivalente au $\mathrm{XIX}^{\mathrm{e}}$ siècle à ce qu'on a appelé l' "ironie romantique », tandis que celle dont les actants sont finalement localisables et stables, qui est donc finalement stabilisée, correspondrait à l' « ironie classique "; la dernière est marquée plus explicitement par la fonction critique - négative, notamment des valeurs sociales, et dotée d'une finalité pédagogique. L'ironie romantique a un caractère moins ouvertement axiologique, et s'inscrit plutôt dans un projet heuristique et épistémologique de mise en doute de nos moyens de perception et de connaissance ; selon la distribution de l'ironie et le niveau de la résolution ou non de la tension dans le texte on distingue entre des effets délimités d'ironie locale comme dans l'exemple cité, ou, lorsqu'elle constitue une posture d'énonciation, d'une ironie globale ${ }^{8}$.

Quant au discours fantastique, il s'agit aussi d'un discours double et paradoxal, puisqu'il doit rendre possibles des explications incompatibles; nous proposerons une analyse selon laquelle il s'articule lui - aussi sur la tension entre deux niveaux : le deuxième niveau, celui du fantastiquesurnaturel, se greffe au premier niveau, celui du réel, en relation anti-thétique, c'est-à-dire citationnelle et polémique comme on l'a décrit pour le discours ironique ; en effet, seule

${ }^{7}$ V. aussi le "principe de pertinence» dans la Relevance Theory de Sperber - Wilson, le "maxime de relation " chez Grice, la "norme » chez Berrendonner, la "doxa" chez Barthes et la notion plus interactive de "l'univers de croyance " de R. Martin, (R. Martin, "Irony and universe of belief », Lingua 87, 1992, pp. 77-90).

${ }^{8}$ On exploite les catégories d'ironie (stable/unstable, local/infinite, covert/overt) de W. Booth, A Rhetoric of Irony, Chicago, University of Chicago Press, 1974, p. 235, qui sont purement rhétoriques, en les intégrant dans une problématique sémio-poétique et historique ; on prime une approche dynamique basée sur la notion de la "tension", inhérente dans l'ironie. 
la constitution d'un espace réaliste permet l'effraction transgressive du fantastique, lequel inscrit le discours réaliste comme dominante et nécessité structurelle9.

L'effet fantastique est créé par la tension entre deux normes, celle de la pertinence naturelle, conforme à l' " encyclopédie », le "connu » dans un monde réel (ici, que « la Mort est une étape absolue et irrévocable ») et celle de la pertinence surnaturelle qui survient dans ce monde réel, et qui est celle des morts-vivants ; c'est précisément leur coprésence paradoxale qui les qualifie de "normes d'impertinence » par rapport à une "norme de pertinence " fantas tique, dans le double sens du mot, qui saurait donner une version adéquate des faits tout en tenant compte à la fois des deux normes paradoxales; leur tension doit aboutir à une certaine déstabilisation - discrédit de la norme de la rationalité chez le lecteur et le personnage qui subit l'effet fantastique, le "fantastisé »- terme proposé par analogie au terme « ironisé »; néanmoins, en même temps, le surnaturel ne doit pas être davantage accrédité. L'effet fantastique advient précisément grâce à la coexistence paradoxale et angoissante de deux normes, par l'indécidabilité qui perpétue la tension.

La thématique de la "morte-vivante » nous introduit au domaine de l'ambiguïté fondatrice de l'ironique et du fantastique et constitue une belle allégorie de leur structure double et antithétique. A l'origine, mort-vivant et Double se confondent puisque dans la mentalité primitive chaque homme possède un double immortel, séparé de lui au moment de la mort ${ }^{10}$. Le Double, thème-clé du fantastique répercute la rupture du principe de l'Identité sous ses deux formes : comme unité rompue par la multiplication externe, le redoublement, et comme intégrité brisée par scission interne, le

${ }^{9}$ Cf. aussi C. LEROY, «Limites non - frontières entre humour et fantastique dans l'écriture surréaliste », Europe 611, "Les Fantastiques ", mars 1980, pp. 71-2. Cependant, dans sa conception de l'humour et du fantastique comme des "écritures secondes, parasitaires, greffées », Leroy les réduit au rôle du «double » en leur niant toute autonomie discursive et donc le statut de genre.

${ }^{10}$ V. et J. EHRSAM, La Littérature Fantastique en France, Paris, Hatier, 1985, pp. 58 et 67. 
dédoublement ${ }^{11}$. Ainsi le double peut être un Autre ou un Soi. Toute cette préoccupation est bien propre au XIX ${ }^{\mathrm{e}}$ siècle, où l'Autre, surtout l'Autre en Soi, est découvert ; désormais l'Autre, le Fou, le Monstre, n'est pas fondamentalement différent de nous, comme l'inconnu n'est pas dans le monde extérieur, mais à l'intérieur de l'homme ${ }^{12}$. Cette inversion de perspective, constitue, on pourrait dire, le côté « ironique » du fantastique. Bien plus, puisqu'au sein du discours fantastique il y a une tendance qui veut ramener la dualité schizoïde du fantastique à l'Unité perdue, le fantastique s'apparente à l'ironie romantique, qui au XIX ${ }^{\mathrm{e}}$ siècle est essentiellement tragique à cause de cette quête d'unité vouée à l'échec ${ }^{13}$. A l'intérieur du Double s'instaurent ainsi des rapports de force, polémiques, pour ramener le Double à l'Unité originaire via la possession et l'aliénation entreprise par la partie qui se greffe sur l'autre; cette relation polémique entre Soi et Double s'illustre dans les nouvelles fantastiques comme dans l'exemple classique du "Horla » de Maupassant ou l'effet final de possession du corps mourant de Rowena par la revenante Ligeia dans la nouvelle homonyme de Poe; ou bien encore la possession du corps du comte d'Athol par Véra : Il ne pouvait que la trouver toujours présente, tant la forme de la jeune femme était mêlée à la sienne [...] D'Athol vivait double, en illuminé (ibid., pp. 557-8).

Les mêmes principes de dualité ou pluralité polémique forment aussi le discours ironique ; celui-ci est un discours polyphonique ou au moins discours du Double, de l'Autre en Soi ; on retrouve l'Autre en Soi, en tant que citation de la parole de l'Autre dans son propre discours et dans la prise en charge énonciative de sa parole, ce qui marque l'ironie comme un discours de possession, appropriation et aliénation du

$11 \mathrm{Cf}$. J. FABRE, Le miroir de sorcière: essai sur la littérature fantastique, Paris, José Corti, 1992, pp. 235-6. 36.

12 Cf. J. MALRIEU, Le fantastique, Paris, Hachette, 1992, pp. 20-

13 Pour la relation du tragique et du fantastique au XIX $\mathrm{X}^{\mathrm{e}}$ siècle, cf. J. Fabre, op. cit., pp. 438 sqq. 
discours de l'Autre ${ }^{14}$; on pourrait ainsi parler du côté «fantastique » du discours ironique.

\section{Une idéologie subversive}

La différence du fantastique par rapport à l'ironie - son statut relevant davantage d'une ambiguitté au domaine intellectuel - est le manque d'axiologie explicite, sa finalité pragmatique étant plutôt l'«angoisse » du lecteur que la "critique »; la dernière est l'apanage de toute ironie et par excellence de l'ironie classique; le fantastique s'apparente plutôt au projet heuristique de l'ironie romantique où l'axiologie est moins forte. Néanmoins puisque «toute ambition de connaissance demande qu'on règle ses comptes avec un interdit et une menace $»^{15}$, le fantastique puise aussi sa thématique au croisement des normes - tabous et surtout de celles de la sexualité et de la mort.

La fonction du deuxième niveau dans le fantastique, comme dans l'ironique est ainsi la transgression des normes discursives et sociales. Il s'agit donc de discours « impertinents » à double objectif ; « impertinents » puisqu'ils visent à la subversion de la "bienséance » du discours qui exige la non-agression du co-locuteur (ce que contourne l'ironie par la citation polémique de la parole d'autrui) et le respect des tabous (ce qui est esquivé par le fantastique et parfois par l'ironie aussi). Les deux discours sont «impertinents » aussi dans le sens qu'ils mirent l'instauration d'une autre pertinence par la transgression des "normes de pertinence » que créent les clichés et les tabous du discours réaliste. En ce qui concerne cette autre norme de Pertinence, plus ou moins explicite dans l'ironie classique, elle reste bien ambiguë dans l'ironie romantique et le fantastique, puisque le projet heuristique de la première, qui concerne la possibilité $\mathrm{du}$ savoir en disqualifiant tous les moyens à la fois, s'apparente bien à celui du fantastique où « [...] il n'est pas

14 Pour l'enjeu de l'identité du sujet par la présence de l'autre dans l'énonciation polyphonique, cf. J. Authier - Revuz, "Hétérogénéité(s) Enonciative(s) ", Langages 73, 1984, pp. 98-111.

15 G. BENREKASSA, "Écrire, penser ; écrire, connaître », Europe 849-50, "Littérature \& philosophie », Jan-Fev. 2000, p. 265. 
question de connaître ni de comprendre le monde, puisque le $\mathrm{F}$ [antastique] implique la disparition même de la possibilité de rendre raison du monde ${ }^{16}$.

Le thème du «mort-vivant » est d'ailleurs un thème emblématique du genre ; issu de la peur, et voire de celle de la mort, source première du fantastique, ce thème se prête aussi à la frayeur par l'abolition des distinctions logiques rigides; la mort est vue comme loi naturelle du monde, et en ce sens les morts-vivants sont en rupture avec la norme de Pertinence. Dans « Véra » et «Ligeia » la norme d'impertinence visée est celle du tabou et du cliché pour la Mort «comme étape absolue et irrévocable »; dans l'exemple d'ironie locale chez "Véra », la norme de Pertinence proposée par le Fantastique et l'Ironique dans la phrase "Puisqu'elle se croit morte» est la conviction que "Véra n'est pas morte ", ou même à l'échelle de la nouvelle fantastique, que «la mort n'est qu'une question de foi, de Véra », selon la signification foi du nom Véra en russe. Pourtant au niveau de l'énonciation ironique globale de la nouvelle qui est celui de l'ironie romantique, l'évanescence de l'apparition surnaturelle de Véra mais la présence mystérieuse de la clef du tombeau dans la chambre rendent cette norme de pertinence impertinente, sans pour autant que le texte nous en procure une ultime norme de Pertinence ; cela perpétue la tension et rallie l'effet ironique à l'effet fantastique.

\section{Effet, réception et coexistence du fantastique et de l'ironique}

\section{Distanciation Comique et Identification Fantastique}

On conçoit l'effet fantastique plutôt comme un produit de rupture ${ }^{17}$, de transgression de la norme de Pertinence, ou pour reprendre la formulation de Poulet concernant le ridicule,

${ }^{16}$ R. Bozetto, A. Chareyre - MÉJAN, P. et R. Pujade, « Penser le Fantastique ", Europe 611, op. cit., p. 30.

${ }^{17} \mathrm{Cf}$. entre autres M. SCHNEIDER, Histoire de la littérature fantastique en France, Paris, Fayard, 1985, p. 8, P. - G. CASTEX, Le Conte fantastique en France de Nodier à Maupassant, Paris, José Corti, 1951, p. 8 et R. Caillois, Au cour du fantastique, Paris, Gallimard, 1965, p. 161 . 
comme «ce qui est perçu comme un hiatus incongru dans la ligne continue de notre expérience coutumière ${ }^{18}$; cette conception du fantastique rencontre celle du comique sauf que dans le dernier la rupture concerne l'objet, tandis qu'au fantastique, comme on a vu au thème du Double, elle concerne le sujet ; de plus, tandis que le comique se déclenche plutôt par la perception d'une brisure éphémère et locale au milieu d'un monde durable et normal, la faille fantastique exige une maturation progressive, une durée pour se déployer. Ainsi chez le comique a-t-elle un caractère bref, extérieur, qui résulte à la distanciation et la sécurisation du lecteur ; au contraire, dans le fantastique elle provoque une certaine déstabilisation puisque la rupture concerne le sujet, et du fait nécessite une certaine identification du lecteur au personnage qui subit la rupture, le fantastisé. Cette identification du lecteur à la conscience en scission et en tension du fantastisé conduit à un "effet d'inconfort » du premier qui mue de l' « inconfortable » à l'« angoissant »; elle est l'effet d'une écriture qui n'accepte pas d'emblée et tranquillement la solution surnaturelle, comme est le cas du Merveilleux. Même quand le fantastique opte pour celle ci, le lecteur est laissé dans un état de perplexité, une acceptation qui n'est pas exempte de refus; nous pensons que l'effet d' « angoisse » psychique de cette « acceptation-refus » est plus important pour le fantastique, que l' «hésitation» intellectuelle que prône Todorov entre solution logique et surnaturelle ${ }^{19}$; ou plutôt, l' « hésitation » intellectuelle, qui pourrait aussi être l'effet d'une posture d'ironie romantique, se double dans le fantastique d'une angoisse psychique.

Cependant le comique et le fantastique ne sont pas incompatibles au sein de la même nouvelle ${ }^{20}$. En ce qui

18 G. POULET, Études sur le temps humain, t. 1, Paris, Plon, 1950, p. 82 .

19 T. TODOROV, Introduction à la littérature fantastique, Paris, Seuil, 1970, p. 29 sqq.

${ }^{20} \mathrm{Cf}$. l'approche du comique et du poétique en termes de désengagement et d'engagement par J. Cohen, "Comique et poétique ", Poétique 61, 1985, pp. 49-61, où néanmoins la coexistence du comique et du poétique est exclue. 
concerne les manifestations locales, par exemple, au début du récit l'humour ou l'ironie renforcent le fonctionnement du fantastique en donnant du poids au réalisme, en lestant sa sécurisation préalable à l'avènement du surnaturel; chez "Véra », avant l'apparition surnaturelle de celle-ci, le comte d'Athol s'ingénie d'établir des rapports « naturels » entre lui et son épouse morte qui vont jusqu'au jeu et la plaisanterie : Une fois, d'Athol la sentit et la vit si bien auprès de lui, qu'il la prit dans ses bras: mais ce mouvement la dissipa. - «Enfant!» murmura-t-il en souriant. Et il se rendormit comme un amant boudé par sa maîtresse rieuse et ensommeillée [ibid., p. 558].

Évidemment dans ce jeu d'identification - distanciation, le comique met l'accent sur la distanciation par l'effet de sécurisation, selon un schéma inverse de celui du fantastique. De même le pouvoir fantaisiste du comique - la capacité de mettre la réalité entre parenthèses - pourrait nuire au réalisme nécessaire au fantastique comme toile de fond. Cependant le réalisme le plus « sec », qui paraîtrait le plus efficace, présente le danger de la distanciation trop facile du lecteur. Tout semble ainsi être une question de degré qui détermine la dominante du texte, fantastique ou comique, puisque cette alternance jusqu'à un certain point nécessaire, entre sécurisation et peur, distanciation et identification, comique et fantastique, pourrait bien tourner le récit au ridicule ${ }^{21}$. Partant dans la nouvelle fantastique du XIX ${ }^{\mathrm{e}}$ siècle le comique peut coexister avec le fantastique seulement lorsqu'il est limité à des occurrences relativement brèves, dispersées et bien délimitées.

\section{Tension Ironique}

L'ironie, en revanche, comme le fantastique, est un phénomène tenant lieu à l'intérieur du sujet, vu qu'elle nécessite une certaine identification, même feinte et provi-

21 Le thème du «mort-vivant » se prête aussi facilement à la caricature et l'humour noir, comme dans les nouvelles «Fleurs de ténèbres » et "A s'y méprendre ! » de Villiers, ou «The Facts in the case of M. Valdermar» de Poe, atteignant le burlesque dans les nouvelles «A Predicament » et «Some Words with a Mummy ». 
soire, de l'ironiste (et du fait du lecteur co-ironiste aussi) avec l'ironisé ; néanmoins la distanciation finale dans l'ironie classique, s'apparente à celle du comique, qui est destructive de l'effet fantastique. Par contre les effets d'ironie locale qui contribuent à l'ambiguïté et à la tension comme dans "Véra ", de même que l'ironie romantique qui, maintenant la tension jusqu'à la fin, n'arrive pas à une distanciation totale et claire, semblent plus compatibles avec le fantastique.

Le discours ironique partage, du moins provisoirement, l' " effet d'inconfort ", de tension et d'hésitation intellectuelle entre deux niveaux de sens mais n'aboutit pas à une « angoisse » psychique. Celle-ci est propre au fantastique, vu que l'existence de deux niveaux est facile à percevoir et la difficulté consiste plutôt à les accepter; le lecteur doit joindre la position de l'inconfort du «fantastisé », celui qui subit l'effet fantastique et qui demeure en position de tension. Au contraire, l'ironie pose plus de difficultés de perception que d'acceptation, demandant l'identification du lecteur avec l'ironiste, et pas avec l'ironisé ; le principal effet ironique dans l'ironie classique serait plutôt celui de la « complicité réconfortante » du lecteur avec l'ironiste dans sa position de distanciation ; l'inconfort ne se présente que dans la mise en question des doxa, des clichés de la pensée à la manière de Socrate, ou s'il s'agit de plonger le lecteur dans la déstabilisation d'une ironie romantique globale, qui comme posture d'énonciation non localisable, a quelque chose de " fantastique ».

En ce qui concerne leur coexistence à l'échelle globale, on pourrait dire que l'ironique se rallie pleinement avec le fantastique au dernier stade de la lecture pour créer un "effet d'inconfort ultime » qui va au-delà de l'ambiguïté ; le lecteur du fantastique est celui qui peut aussi «comprendre » et non pas simplement «subir », et donc celui qui peut se distancier finalement du fantastisé; ce recul demande une vision ironique, la lucidité est gagnée au prix de l'inversion ironique de la problématique fantastique : le lecteur reconnaît la part du phénomène fantastique qui est en nous, l'autre qui est en nous, qu'en fait c'est nous qui sommes le phénomène fantastique. C'est d'Athol qui vivait en double et le lecteur aussi, le temps d'une lecture. 
Ou bien, suivant le chemin inverse, l'ironie constitue un " effet de réconfort », de sécurisation par rapport au fantastique ; à la fin de la lecture du texte fantastique, le recours à l'ironie et à l'humour semble un mécanisme presque inconscient du lecteur pour se défendre de l'effet envoûtant de celui-ci ; prenant d'abord une distanciation admirative devant l'habileté du conteur à créer une ambiance, il peut passer au sentiment quelque peu nauséabond d'avoir été « eu » dans le double registre du mot, "possédé » par l'effet fantastique et « trompé » par l'effet ironique ; contre quoi il se défend par l'attribution de l'humour et de l'ironie au texte fantastique. Évidemment on ne pourrait pas considérer que la lecture d'un texte fantastique nous introduit d'emblée à un jeu humoristique - ironique de l'auteur. S'il n'y a pas d'indications textuelles - extratextuelles d'une intention ironique, l'interprétation ironique semble arbitraire.

Néanmoins il existe des auteurs de nouvelles fantastiques qui rallient le fantastique et l'ironique, ce qui rend finalement la nouvelle fantastique un " canular ». Il s' agirait ainsi d'un " pacte humoristique » entre écrivain et lecteur comme celui décrit par Poe dans un compte rendu d'un texte fantastique, le roman Sheppard Lee: "L'auteur qui n'est pas tenu de fournir une explication sur les invraisemblances de son récit, a soin de leur conférer le caractère et la clarté de la véracité [...]. Le lecteur également perçoit aisément l'humour de l'écrivain, il s'y rallie et, de ce fait, il consent à se laisser prendre $[\ldots] »^{22}$. L'auteur du récit fantastique ferait donc un pacte ironique et convierait le lecteur à entrer dans son jeu de posture d'énonciation à la fois fantastique et ironique ; son ironie divise le lectorat en deux, ceux qui passent au second degré de la lecture, et ceux qui restent au premier, les lecteurs fantastisés qui sont ainsi en même temps des lecteurs ironisés ; tel est le cas de Poe, dont "Ligeia » ainsi que d'autres " gothic tales » à intention ironique (selon sa correspondance) ont mystifié un grand nombre de son lectorat. Effectivement au cas où l'intention fantastique de l'écrivain ne passerait pas

22 E. A. POE. «Le fantastique : Robert M. Bird», compte rendu de Sheppard Lee (1836) in Edgar Allan Poe, L'Herne 26, C. Richard, (ed.), 1998, pp. 111-2. 
à un niveau ironique, le pacte ironique tel qu'il est décrit par Poe, ne serait qu'un processus qui ressemblerait trop au célèbre « willing suspension of disbelief » de Coleridge qui est à la base du "pacte de la fiction » en général. Il n'empêche que du moment où est primée clairement une lecture ironique l'effet fantastique est en grande partie détruit, sauf dans le cas de l'ironie romantique qui perpétue la tension et l'indécidabilité jusqu'à la fin, comme dans «Véra"; néanmoins l'angoisse psychique du fantastique recule devant l'indécidabilité intellectuelle de l'ironique.

Avec cette analyse tensionnelle de l'ironie et du fantastique, s'appuyant sur leurs affinités structurelles et idéologiques, nous proposerions la conception du fantastique dans la nouvelle du XIX ${ }^{\mathrm{e}}$ siècle comme un discours qui s'apparente fortement - si ce n'est qu'une forme affiliée - à l'ironie tensionnelle (romantique) en tant que posture d'énonciation. Outre leur origine commune chez les romantiques allemands, ils s'entrecoupent à bien des points : la structure double et tensionnelle, la finalité subversive et heuristique, la crise tragique du sujet et l'effet de déstabilisation du lecteur visant plutôt l'angoisse dans le fantastique et l'hésitation intellectuelle dans l'ironique. Nous proposerions aussi la coanalyse fructueuse du Fantastique et de l'Ironique, notamment leur étude en termes d'effets de «confort» et d'« inconfort » par rapport à la distanciation - identification du lecteur, ainsi que l'exploration dans leur schéma communicationnel des notions de "norme de Pertinence-Impertinence » et du «Fantastisé ».

En terminant notre problématique engendrée par le thème de la "morte-vivante ", Véra et Ligeia, on se demande si le discours ironique et le fantastique, se greffant sur le discours réaliste en tant que discours de clichés, le mettant en question et l'aliénant, ne sont pas à leur tour des discours «morts - vivants »; et l'ironique, en tant que posture globale dans la nouvelle fantastique, se fondant et se confondant avec le discours fantastique, s'établissant finalement comme discours ultime, ne devient-il pas forcément à son tour « vampirique », c'est-à-dire fantastique ? 